\title{
Associated $\mathrm{Z}$ boson production in the forward region
}

\author{
Marek Sirendi on behalf of the LHCb collaboration* \\ University of Cambridge \\ E-mail: sirendi@hep.phy.cam.ac.uk
}

\begin{abstract}
A measurement of the $\mathrm{Z}\left(\rightarrow \mu^{-} \mu^{+}\right)+$jet production cross-section and an observation of associated production of a $\mathrm{Z}$ boson with a $\mathrm{D}$ meson in pp collisions at a centre-of-mass energy of $\sqrt{s}=7 \mathrm{TeV}$ is presented. The measurements are based on an integrated luminosity of $1.0 \mathrm{fb}^{-1}$, collected by the $\mathrm{LHCb}$ experiment. For $\mathrm{Z}+$ jet, the overall cross-section in a fiducial volume as well as normalised differential cross-sections are measured for jet transverse momentum thresholds of 10 and $20 \mathrm{GeV}$. For $\mathrm{Z}+\mathrm{D}$, overall cross-sections are measured for $\mathrm{Z}\left(\rightarrow \mu^{-} \mu^{+}\right)+\mathrm{D}^{0}$ and $\mathrm{Z}\left(\rightarrow \mu^{-} \mu^{+}\right)+\mathrm{D}^{+}$production based on eleven candidates in total.
\end{abstract}

XXII. International Workshop on Deep-Inelastic Scattering and Related Subjects, 28 April - 2 May 2014

Warsaw, Poland

* Speaker. 


\section{Introduction}

The LHCb detector [1] is a single-arm forward spectrometer optimised to study b-physics. It covers a pseudorapidity, $\eta$, range of $2.0<\eta<5.0$ making electroweak measurements at LHCb complementary to those performed by the ATLAS and CMS experiments. Here, we report on cross-section measurements of $\mathrm{Z}$ plus jet [2] and $\mathrm{Z}$ plus $\mathrm{D}$ [3] which are performed with the LHCb detector at $\sqrt{s}=7 \mathrm{TeV}$ using $1.0 \mathrm{fb}^{-1}$ of integrated luminosity collected in 2011.

Measurements of electroweak bosons in the forward region can be used to constrain parton distribution functions (PDFs) as they have a unique sensitivity to high and low Bjorken- $x$. Z + jet $^{1}$ events are typically produced from a collision of a sea quark, or a gluon, with a valence quark and are, thus, sensitive to the gluon PDF. The measurement of this process can also be used to tune event generators. On the other hand, $\mathrm{Z}+\mathrm{D}$ is sensitive to the charm PDF, the charm production mechanism, and to double-parton scattering (DPS).

Theoretical predictions for $\mathrm{Z}+$ jet are available at next-to-leading-order (NLO) in the stronginteraction coupling parameter $\alpha_{s}$ and different PDF sets are compared. For $\mathrm{Z}+\mathrm{D}$, theory predictions are computed at LO and NLO for single-parton scattering (SPS), while a factorisation approximation is used to obtain an estimate for DPS.

\section{Event selection}

The fiducial volume of the muons originating from the decay of the $\mathrm{Z}$ is identical for both measurements. Both final state muons are required to have a transverse momentum of at least $20 \mathrm{GeV}$, and lie within a pseudorapidity range of $2.0<\eta^{\mu}<4.5$. The dimuon invariant mass is required to be in the range $60<M_{\mu \mu}<120 \mathrm{GeV}$.

For $\mathrm{Z}+$ jet, the jets are similarly required to be within $2.0<\eta^{\text {jet }}<4.5$ and are reconstructed with the anti- $k_{\mathrm{T}}$ algorithm [4] with a radius parameter of $R=0.5$. The inputs to the jet finding are selected using a particle flow approach. The jets are further required to be well-separated from the $\mathrm{Z}$ such that $\Delta r(\mu, j e t)>0.4 .^{2}$ Measurements are performed for two jet transverse momentum thresholds: 10 and $20 \mathrm{GeV}$. Track quality and jet quality requirements are imposed in the analysis. The measurements are corrected using efficiencies determined from data in the case of muons and from simulation for jets.

In the measurement of $\mathrm{Z}+\mathrm{D}$, the $\mathrm{D}$ candidates are reconstructed through the $\mathrm{D}^{0} \rightarrow \mathrm{K}^{-} \pi^{+}$and $\mathrm{D}^{+} \rightarrow \mathrm{K}^{-} \pi^{+} \pi^{+}$final states. The open charm mesons are reconstructed in a kinematic range of $2<y(\mathrm{D})<4$ and $2<p_{\mathrm{T}}<12 \mathrm{GeV}$. Further kinematic and identification cuts are applied on the daughters of the charm mesons. The selection imposed on the final state muons and the D mesons defines the fiducial volume of the measurement, while the cuts on the kaons and pions are corrected for.

\footnotetext{
${ }^{1}$ The virtual photon $\left(\gamma^{*}\right)$ contribution is always implied.

${ }^{2} \Delta r^{2} \equiv \Delta \phi^{2}+\Delta \eta^{2}$, where $\Delta \phi$ is the difference in azimuthal angle and $\Delta \eta$ is the difference in pseudorapidity between the jet and the $\mathrm{Z}$.
} 


\section{Cross-section measurement}

In the $\mathrm{Z}+$ jet measurement, migrations in the jet transverse momentum are corrected for by unfolding the $p_{\mathrm{T}}^{\text {jet }}$ distribution. Reconstruction and selection efficiencies are computed differentially and the measurement is corrected for these. The dominant systematic uncertainty in the measurement derives from jet reconstruction and the uncertainty on the jet energy scale. The measurement is quoted at the Born level in QED using correction factors derived from theory for final state radiation (FSR) of the muons.

In the study of $\mathrm{Z}+\mathrm{D}$, the $\mathrm{Z}$ boson and the $\mathrm{D}$ meson are required to originate from the same $\mathrm{pp}$ interaction and the contribution from multiple pp interactions is quantified. Open charm mesons that originate from beauty hadrons are considered background. A two-dimensional fit to the mass distributions of the $\mathrm{Z}$ and the $\mathrm{D}$ candidates is performed to quantify the contribution of the combinatorial background and to assess the significance of the observation. The total purity, defined as the fraction of signal in the selected sample, is determined to be above $95 \%$ for both $\mathrm{Z}$ plus $\mathrm{D}^{0}$ and $\mathrm{Z}$ plus $\mathrm{D}^{+}$with the dominant background contribution coming from b-quark decays.

Pseudo-experiments are carried out whereby the significance can be extracted. In each such experiment, events are sampled according to a background-only hypothesis. The resultant distributions are fit using probability distribution functions describing the signal and a uniform distribution to describe the combinatorial background. The combinatorial background is best described by an exponential function, while the use of a uniform distribution results in a conservative estimate of signal significance. A significance of 3.7 and 3.3 standard deviations is obtained for the $\mathrm{D}^{0}$ and $\mathrm{D}^{+}$cases, respectively. Finally, the combined significance of $\mathrm{Z}+\mathrm{D}$ production corresponds to 5.1 standard deviations.

\section{Results}

For $\mathrm{Z}+$ jet, the total cross-section as well as the cross-section ratio to inclusive $\mathrm{Z}$ production is reported. In addition, differential measurements as a function of the $\mathrm{Z}$ boson rapidity and transverse momentum, the pseudorapidity and transverse momentum of the leading jet, and the difference in azimuthal angle and in rapidity between the $\mathrm{Z}$ and this jet are reported. The differential measurements are normalised to the total $\mathrm{Z}+$ jet cross-section.

For the $20 \mathrm{GeV}$ jet transverse momentum threshold, the cross-section and cross-section ratio are measured to be

$$
\begin{aligned}
& \sigma\left(\mathrm{Z}\left(\rightarrow \mu^{-} \mu^{+}\right)+\text {jet }\right)=6.3 \pm 0.1 \text { (stat.) } \pm 0.5 \text { (syst.) } \pm 0.2 \text { (lumi.) } \mathrm{pb}, \\
& \frac{\sigma\left(\mathrm{Z}\left(\rightarrow \mu^{-} \mu^{+}\right)+\text {jet }\right)}{\sigma\left(\mathrm{Z}\left(\rightarrow \mu^{-} \mu^{+}\right)\right)}=0.083 \pm 0.001 \text { (stat.) } \pm 0.007 \text { (syst.) }
\end{aligned}
$$

while for the $10 \mathrm{GeV}$ threshold one obtains,

$$
\begin{aligned}
& \sigma\left(\mathrm{Z}\left(\rightarrow \mu^{-} \mu^{+}\right)+\text {jet }\right)=16.0 \pm 0.2 \text { (stat.) } \pm 1.2 \text { (syst.) } \pm 0.6 \text { (lumi.) } \mathrm{pb}, \\
& \left.\frac{\sigma\left(\mathrm{Z}\left(\rightarrow \mu^{-} \mu^{+}\right)+\text {jet }\right)}{\sigma\left(\mathrm{Z}\left(\rightarrow \mu^{-} \mu^{+}\right)\right)}=0.209 \pm 0.002 \text { (stat. }\right) \pm 0.015 \text { (syst.) }
\end{aligned}
$$

where the first uncertainty is statistical, the second is systematic, and the third is the uncertainty due to luminosity determination. 


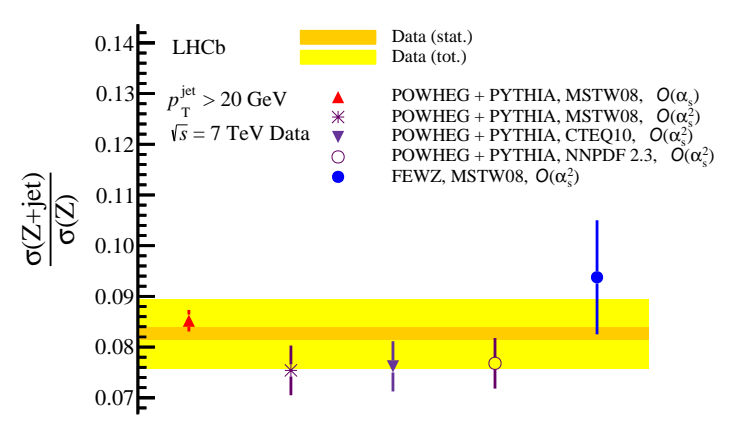

(a) $\sigma(\mathrm{Z}+$ jet $) / \sigma(\mathrm{Z})$ for $p_{\mathrm{T}}^{Z}>20 \mathrm{GeV}$

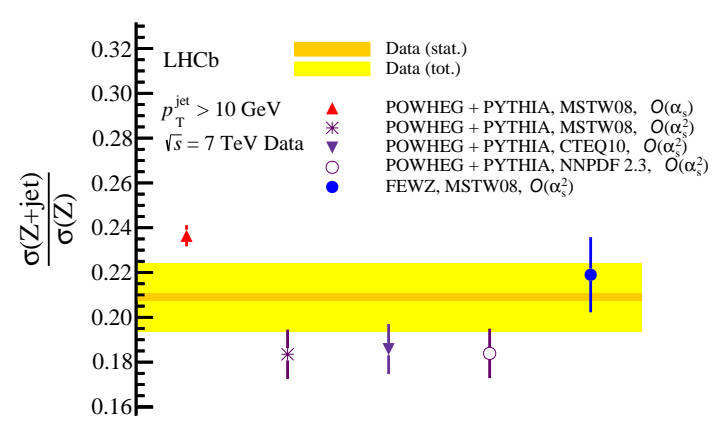

(b) $\sigma(\mathrm{Z}+$ jet $) / \sigma(\mathrm{Z})$ for $p_{\mathrm{T}}^{Z}>10 \mathrm{GeV}$

Figure 1: Ratio of the $\mathrm{Z}+$ jet cross-section to the inclusive cross-section for two momentum thresholds. The bands give the LHCb measurement corrected for FSR with the statistical uncertainty (inner band) and the total uncertainty (outer band). The points show the theory predictions with uncertainties as defined in the main text.

Theoretical predictions are computed at NLO using POWHEG [5-8] with PYTHIA [9] simulation of parton shower development and hadronisation. An uncertainty on these predictions is obtained by varying the renormalisation and factorisation scales around the value of the vector boson transverse momentum. In computing theory predictions, three NLO PDF parameterisations are compared: MSTW08 [10], CTEQ10 [11] and NNPDF 2.3 [12]. A leading-order MSTW08 PDF parameterisation is also contrasted. For the cross-section ratio measurement, the inclusive $\mathrm{Z}$ cross-section is computed at leading-order for the MSTW08, CTEQ10, and NNPDF 2.3 PDF parameterisations. Furthermore, a separate theory prediction is computed using FEWZ [13] which neglects the effects from hadronisation and the underlying event. In this case, the renormalisation and factorisation scales are set to the nominal value of the vector boson mass.

Cross-section ratios are shown in Fig. 1 while normalised differential cross-sections, uncorrected for final state radiation from the muons, are displayed on Figs. 2-4. Reasonable agreement is observed in all variables for the $\mathscr{O}\left(\alpha_{s}^{2}\right)$ calculations, while differences are observed with the $\mathscr{O}\left(\alpha_{s}\right)$ calculation for the measurements in $p_{\mathrm{T}}^{j e t}, p_{\mathrm{T}}^{Z}$, and $\Delta \phi$.

The cross-sections for $\mathrm{Z}\left(\rightarrow \mu^{-} \mu^{+}\right)+\mathrm{D}$ are determined to be

$$
\begin{aligned}
& \sigma\left(\mathrm{Z}\left(\rightarrow \mu^{-} \mu^{+}\right)+\mathrm{D}^{0}\right)=2.50 \pm 1.12 \text { (stat.) } \pm 0.22 \text { (syst.) } \mathrm{pb} \\
& \sigma\left(\mathrm{Z}\left(\rightarrow \mu^{-} \mu^{+}\right)+\mathrm{D}^{+}\right)=0.44 \pm 0.23 \text { (stat.) } \pm 0.03 \text { (syst.) } \mathrm{pb}
\end{aligned}
$$

where the first uncertainty is statistical and the second is systematic.

The SPS prediction for the production of Zcc is computed at leading order and at NLO in the massless c-quark approximation while contributions from Zc are obtained at NLO. The kinematic criteria are applied to the c-quark and hadronisation is corrected for in the theory prediction. The DPS cross-section is obtained using a factorisation ansatz based on the effective DPS cross-section [14]. The sum of the predictions for SPS and DPS is consistent with the measured cross-section for $\mathrm{Z}+\mathrm{D}^{0}$ production, while clear tension is observed in the prediction for $\mathrm{Z}+\mathrm{D}^{+}$production (Fig. 5). 


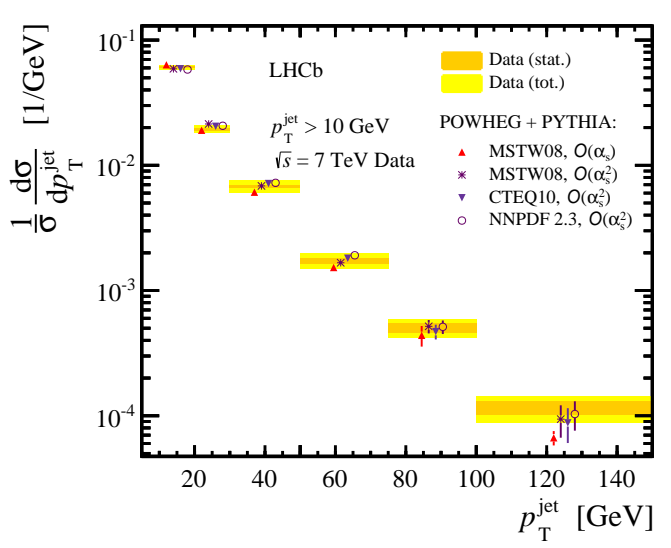

(a) $\frac{1}{\sigma} \frac{\mathrm{d} \sigma}{\mathrm{d} p_{\mathrm{T}}^{j e t}}$ for $p_{\mathrm{T}}^{Z}>10 \mathrm{GeV}$

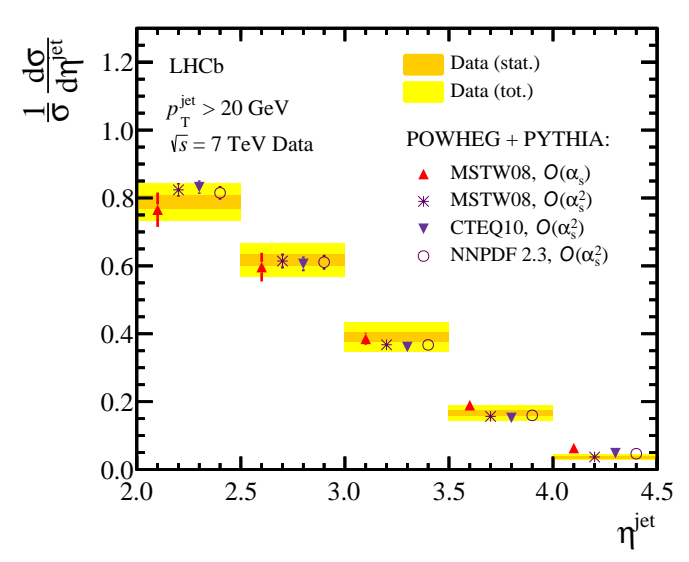

(b) $\frac{1}{\sigma} \frac{\mathrm{d} \sigma}{\mathrm{d} \eta^{j e t}}$ for $p_{\mathrm{T}}^{Z}>20 \mathrm{GeV}$

Figure 2: The differential $\mathrm{Z}+$ jet cross-section as a function of $p_{\mathrm{T}}^{\text {jet }}$ (left) and $\eta_{\mathrm{T}}^{\text {jet }}$ (right). The bands give the $\mathrm{LHCb}$ measurement with the statistical uncertainty (inner band) and the total uncertainty (outer band). The points show the theory predictions with uncertainties as defined in the main text. Predictions are displaced horizontally for presentation. These results have not been corrected for FSR from the final state muons.

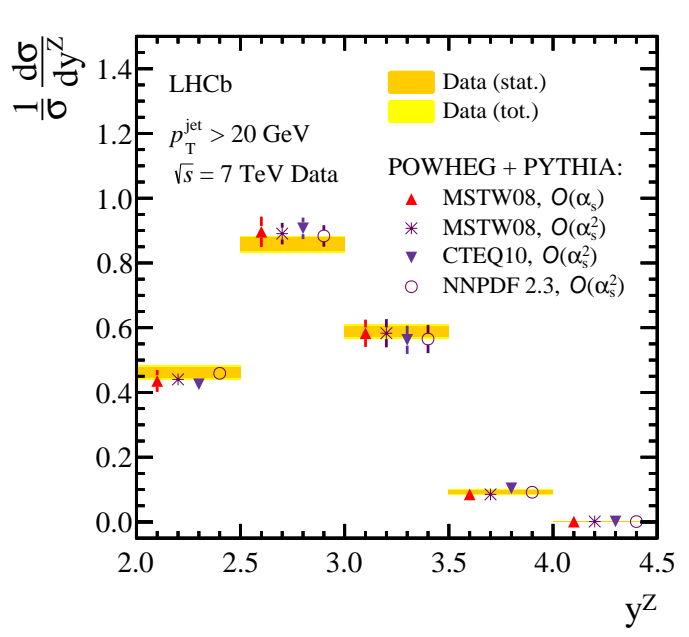

(a) $\frac{1}{\sigma} \frac{\mathrm{d} \sigma}{\mathrm{d} y^{Z}}$ for $p_{\mathrm{T}}^{Z}>20 \mathrm{GeV}$

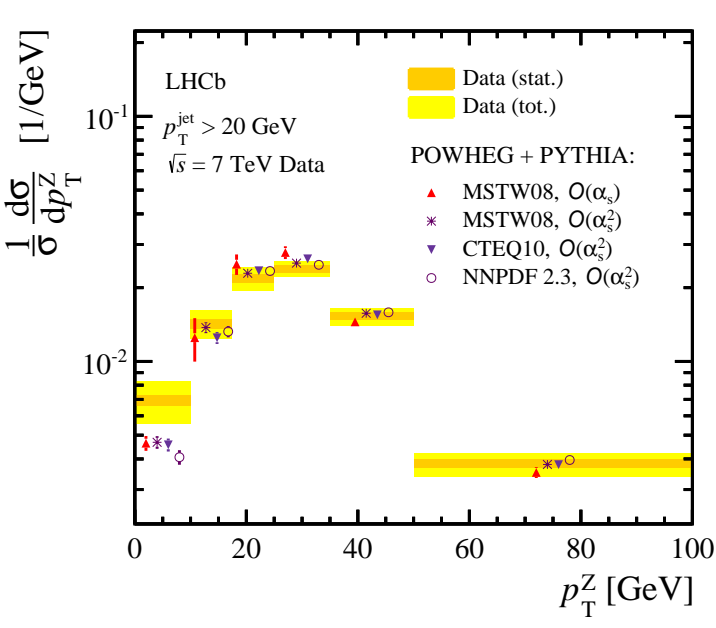

(b) $\frac{1}{\sigma} \frac{\mathrm{d} \sigma}{\mathrm{d} p_{\mathrm{T}}^{Z}}$ for $p_{\mathrm{T}}^{Z}>20 \mathrm{GeV}$

Figure 3: The differential $\mathrm{Z}+$ jet cross-section as a function of $y^{\mathrm{Z}}$ (left) and $p_{\mathrm{T}}^{\mathrm{Z}}$ (right). The bands give the LHCb measurement with the statistical uncertainty (inner band) and the total uncertainty (outer band). The points show the theory predictions with uncertainties as defined in the main text. Predictions are displaced horizontally for presentation. These results have not been corrected for FSR from the final state muons. 


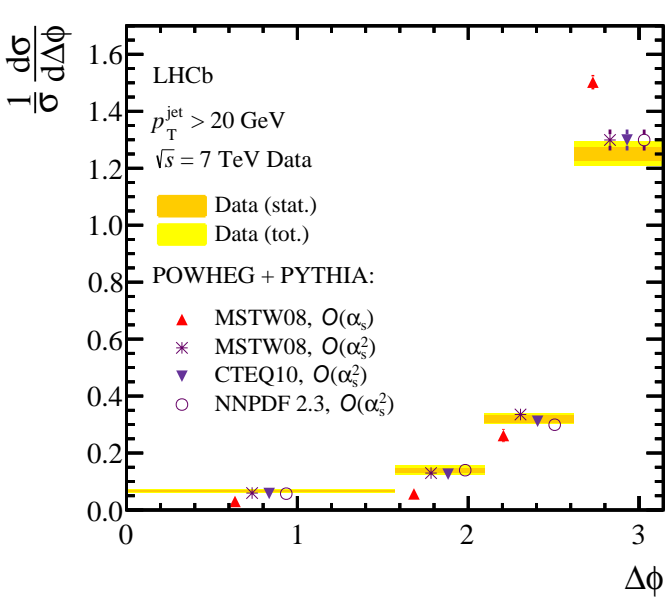

(a) $\frac{1}{\sigma} \frac{\mathrm{d} \sigma}{\mathrm{d} \Delta \phi}$ for $p_{\mathrm{T}}^{Z}>20 \mathrm{GeV}$

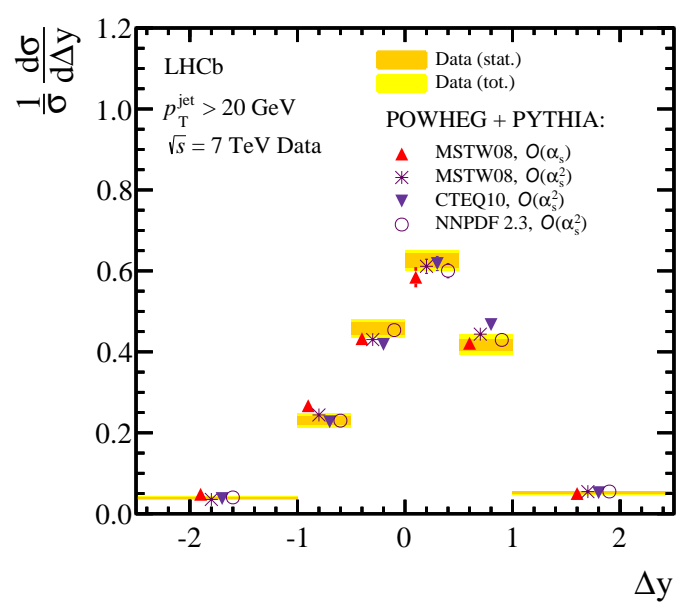

(b) $\frac{1}{\sigma} \frac{\mathrm{d} \sigma}{\mathrm{d} \Delta y}$ for $p_{\mathrm{T}}^{Z}>20 \mathrm{GeV}$

Figure 4: The differential $\mathrm{Z}+$ jet cross-section as a function of $\Delta \phi$ (left) and $\Delta y$ (right). The bands give the $\mathrm{LHCb}$ measurement with the statistical uncertainty (inner band) and the total uncertainty (outer band). The points show the theory predictions with uncertainties as defined in the main text. Predictions are displaced horizontally for presentation. These results have not been corrected for FSR from the final state muons.

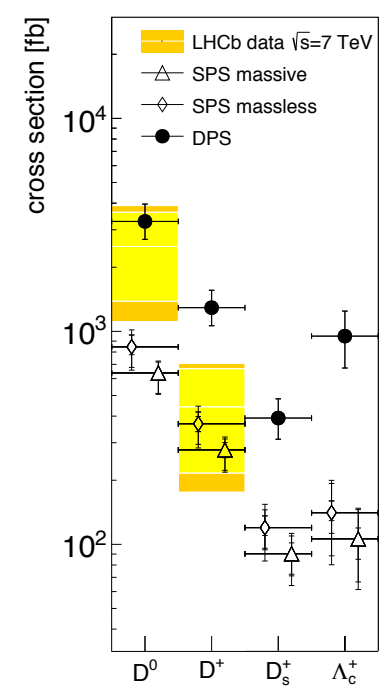

Figure 5: Theory predictions of SPS and DPS as points with the measured cross-sections as bands with the statistical uncertainty (inner band) and the total uncertainty (outer band). SPS predictions are given both in the massless cquark approximation and for a massive c-quark as described in the main text. No $\mathrm{D}_{\mathrm{s}}^{+}$or $\Lambda_{\mathrm{c}}^{+}$candidates were observed.

\section{Summary}

Measurements of inclusive $\mathrm{Z}+$ jet and $\mathrm{Z}+\mathrm{D}$ cross-sections at $\sqrt{s}=7 \mathrm{TeV}$ are presented. For $\mathrm{Z}+$ jet, normalised differential cross-sections are also measured as a function of variables that describe $\mathrm{Z}$ boson and jet kinematic properties. All NLO PDF parameterisations studied show reasonable agreement with the measured results.

Associated production of a $\mathrm{Z}$ with a $\mathrm{D}$ meson has been observed at $\mathrm{LHCb}$ for the first time. With more data a measurement of the differential distributions will be possible, which would enable the SPS and DPS contributions to be disentangled. 


\section{References}

[1] LHCb collaboration, The LHCb detector at the LHC, 2008 JINST 3 S08005.

[2] LHCb collaboration, Study of forward Z + jet production in pp collisions at $\sqrt{s}=7 \mathrm{TeV}$, JHEP 01 (2014) 033.

[3] LHCb collaboration, Observation of associated production of a $\mathrm{Z}$ boson with a $\mathrm{D}$ meson in the forward region, JHEP 04 (2014) 091.

[4] M. Cacciari, G.P. Salam, G. Soyez, The anti- $k_{\mathrm{T}}$ jet clustering algorithm, JHEP 04 (2008) 063.

[5] S. Alioli, P. Nason, C. Oleari and E. Re, Vector boson plus one jet production in POWHEG, JHEP 01 (2011) 095.

[6] P. Nason, A New method for combining NLO QCD with shower Monte Carlo algorithms, JHEP 11 (2004) 040.

[7] S. Frixione, P. Nason and C. Oleari, Matching NLO QCD computations with parton shower simulations: the POWHEG method, JHEP 11 (2007) 070.

[8] S. Alioli, P. Nason, C. Oleari and E. Re, A general framework for implementing NLO calculations in shower Monte Carlo programs: the POWHEG BOX, JHEP 06 (2010) 043.

[9] T. Sjöstrand, S. Mrenna and P.Z. Skands, PYTHIA 6.4 physics and manual, JHEP 05 (2006) 026.

[10] A. Martin, W. Stirling, R. Thorne and G. Watt, Parton distributions for the LHC, Eur. Phys. J. C 63 (2009) 189.

[11] H.-L. Lai et al., New parton distributions for collider physics, Phys. Rev. D 82 (2010) 074024.

[12] R.D. Ball et al., Parton distributions with LHC data, Nucl. Phys. B 867 (2013) 244.

[13] R. Gavin, Y. Li, F. Petriello and S. Quackenbush, FEWZ 2.0: a code for hadronic Z production at next-to-next-to-leading order, Comput. Phys. Commun. 182 (2011) 2388.

[14] CDF collaboration, Double parton scattering in $\bar{p} p$ collisions at $\sqrt{s}=1.8 \mathrm{TeV}$, Phys. Rev. D56 (1997) 3811. 